\section{Serial Electrocardiographic Changes in Fulminant Myocarditis - Reply -}

Thank you for your valuable and encouraging comments on our study. ${ }^{1}$ I totally agree with your comments. As we stated in our paper, ${ }^{1}$ patients with fulminant myocarditis have severe conduction abnormalities or life-threatening arrhythmias in the acute phase, and, along with an improvement in their cardiac function and ventricular wall thickness, these arrhythmias disappear in the convalescent phase. The survival rate from fulminant myocarditis in our series was $57 \%$ overall, and the patients with fulminant myocarditis had a relatively good prognosis if they survived the acute phase. Approximately $30 \%$ of the patients with acute myocarditis were reported to have developed dilated cardiomyopathy (DCM). Fortunately, none of the 4 survivors in our series showed a decrease in ventricular function during the follow up period.

Fulminant myocarditis is caused mostly by the direct invasion of viruses and an increase in cytokines such as interleukin (IL)-1 $\beta$, IL-6, tumor necrosis factor- $\alpha$, and interferon- $\gamma \cdot{ }^{2,3}$ These acute phase changes can destroy the conduction system and also the myocardium, with subsequent occurrence of lethal arrhythmias and acute cardiac failure.

The question has arisen as to whether the damaged conduction system and working myocardium can recover in the convalescent phase. The development of DCM is likely due to continual damage to the ventricular myocardium by an autoimmune or chemical mechanism, and also the loss of a protection mechanism in the myocardial cells per se. Recently, IL-17A was reported to be one of the most important factors contributing to myocardial fibrosis after myocarditis. ${ }^{4}$ The use of monoclonal antibodies for IL-17A in the acute phase of myocarditis may prevent the remodeling due to myocardial fibrosis and also the development of DCM in the future.

\section{References}

1. Ichikawa R, Sumitomo N, Komori A, Abe Y, Nakamura T, Fukuhara $\mathrm{J}$, et al. The follow-up evaluation of electrocardiogram and arrhythmias in children with fulminant myocarditis. Circ J 2011; 75: 930938.

2. Kawai C. From myocarditis to cardiomyopathy: Mechanisms of inflammation and cell death learning from the past for the future. Circulation 1999; 99: 1091-1100.

3. Abe S, Okura Y, Hoyano M, Kazama R, Watanabe S, Ozawa T, et al. Plasma concentrations of cytokines and neurohumoral factors in a case of fulminant myocarditis successfully treated with intravenous immunoglobulin and percutaneous cardiopulmonary support. Circ J 2004; 68: $1223-1226$.

4. Baldeviano GC, Barin JG, Talor MV, Srinivasan S, Bedja D, Zheng D, et al. Interleukin-17A is dispensable for myocarditis but essential for the progression to dilated cardiomyopathy. Circ Res 2010; 106: $1646-1655$.

Naokata Sumitomo, MD, PhD

Department of Pediatrics and Child Health, Nihon University School of Medicine, Tokyo, Japan

(Released online July 12, 2011) 